\title{
NOTE ON THE PREVALENCE OF INTESTINAL WORMS IN DOGS IN CAMBRIDGE.
}

\author{
By GEORGE H. F. NUTTALL AND CYRIL STRICKLAND.
}

IN the course of investigations conducted in Cambridge upon dogs infected with Piroplasma canis, a number of animals have been incidentally examined for intestinal parasites. In an unbroken series of 24 dogs, which died (March-September 1907) from piroplasmosis, the animals were antopsied immediately after death and the intestine examined by slitting it open along its whole length. We briefly record the results of these examinations, for the reason that similar reports do not appear to have hitherto been published in this country. All of the dogs harboured worms. Only three species of worms were encountered : Ascaris mystax, Dipylidium caninum and Taenia serrata.

The parasites were distributed as follows :-

\begin{tabular}{llllc} 
& & \multicolumn{3}{c}{$\begin{array}{c}\text { No. of dogs har- } \\
\text { bouring each species }\end{array}$} \\
A. mystax & $\ldots$ & $\ldots$ & $\ldots$ & 17 \\
D. caninum & $\ldots$ & $\ldots$ & $\ldots$ & 14 \\
T. serrata & $\ldots$ & $\ldots$ & $\ldots$ & 4
\end{tabular}

The species occurred either alone or associated with each other :

10 dogs harboured A. mystax.

$\begin{array}{rlll}5 & & & \text { D. caninum. } \\ 5 & " & & \text { A. mystax and D. caninum. } \\ 2 & " & \text { D. caninum and T. serrata. } \\ 2 & \quad & \text { A. mystax, D. caninum and T. serrata. }\end{array}$

The numbers of $A$. mystax varied from 1 to 113 per dog.

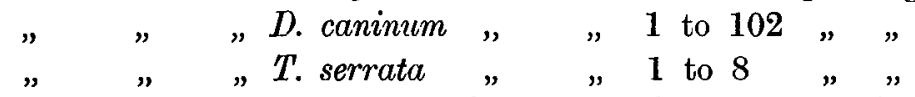

The total number of each species recovered from the 24 dogs was
A. mystax 296.
D. caninum 312.
T. serrata 17. 
The following table contains details regarding the numbers and species of worms found in each of the dogs examined :-

\begin{tabular}{|c|c|c|c|c|c|}
\hline $\begin{array}{l}\text { No. of } \\
\text { dog }\end{array}$ & $\begin{array}{l}\text { Species } \\
\text { of worm }\end{array}$ & $\begin{array}{l}\text { No. of } \\
\text { worms found }\end{array}$ & $\begin{array}{l}\text { No. of } \\
\text { dog }\end{array}$ & $\begin{array}{l}\text { Species } \\
\text { of worm }\end{array}$ & $\begin{array}{c}\text { No. of } \\
\text { worms found }\end{array}$ \\
\hline 1 & A. mystax & 4 & 13 & A. mystax & 3 \\
\hline \multirow{3}{*}{2} & (D. caninum & 1 & 14 & D. caninum & 1 \\
\hline & T. serrata & 3 & 15 & D. caninum & 82 \\
\hline & A. Mystax & 2 & 16 & A. mystax & 2 \\
\hline \multirow[t]{2}{*}{3} & D. caninum & 2 & \multirow{2}{*}{17} & D. caninum & 30 \\
\hline & (T. serrata & 1 & & A. mystax & 6 \\
\hline 4 & A. $\operatorname{mystax}$ & 5 & \multirow{3}{*}{18} & D. caninum & 1 \\
\hline 5 & A. mystax & 1 & & A. mystax & 45 \\
\hline 6 & A. mystax & 48 & & (T. serrata & 5 \\
\hline 7 & A. mystax & 4 & 19 & D. caninum & 1 \\
\hline 8 & D. caninum & 7 & \multirow[t]{2}{*}{20} & D. caninum & 53 \\
\hline \multirow{2}{*}{9} & D. caninum & 20 & & 'A. mystax & 7 \\
\hline & T. serrata & 8 & 21 & A. mystax & 5 \\
\hline \multirow{2}{*}{10} & $\{$ D. caninum & 7 & 22 & A. mystax & 31 \\
\hline & A. mystax & 2 & \multirow{2}{*}{23} & A. mystax & 113 \\
\hline \multirow{2}{*}{11} & IA. mystax & 4 & & ID. caninum & 4 \\
\hline & D. caninum & 1 & \multirow[t]{2}{*}{24} & D. caninum & 102 \\
\hline 12 & A. mystax & 14 & & & \\
\hline
\end{tabular}

